\title{
Perspective Piece \\ Putting Guidance into Practice: Interpretation of the Oral Cholera Vaccine Recommendations for U.S. Travelers
}

\author{
Erin E. Conners, ${ }^{1 *}$ Kristina M. Angelo, ${ }^{2}$ and Allison T. Walker ${ }^{2}$ \\ ${ }^{1}$ Epidemic Intelligence Service Officer Assigned to the Division of Foodborne, Waterborne, and Environmental Diseases, National \\ Center for Emerging Zoonotic and Infectious Diseases, CDC, Atlanta, Georgia; ' $T$ Travelers' Health Branch, Division \\ of Global Migration and Quarantine, CDC, Atlanta, Georgia
}

\begin{abstract}
The American Committee on Immunization Practices recommends the use of the oral cholera vaccine (OCV) in international travelers aged 18-64 years who visit areas of active cholera transmission. CDC Travelers' Health Branch currently tracks areas of active cholera transmission and posts this information on their countryspecific destination web pages at https://wwwnc.cdc.gov/travel/destinations/list. Lessons learned from a webinar conducted among health care providers have led to improvements in how CDC shares information on the OCV. Here, we summarize the major considerations for providers offering the OCV to U.S. travelers and indicate where they can find more information.
\end{abstract}

\section{ORAL CHOLERA VACCINE RECOMMENDATIONS IN THE UNITED STATES}

Cholera, caused by toxigenic strains of the bacteria Vibrio cholerae serogroups $\mathrm{O} 1$ and $\mathrm{O} 139$, may cause severe illness and can be rapidly fatal without treatment. Most cases of cholera reported in the United States occur among international travelers to countries in which cholera is endemic or epidemic. ${ }^{1}$ An oral cholera vaccine (OCV), lyophilized CVD 103-HgR (Vaxchora ${ }^{\circledR}$, PaxVax Bermuda Ltd., Redwood City, CA), has been shown to be safe and effective for the prevention of illness from cholera caused by $V$. cholerae serogroup 01 . In vaccine challenge studies, this OCV reduced severe diarrhea by $90 \%$ at 10 days after vaccination and by $80 \%$ at 3 months after vaccination. ${ }^{2}$

In 2016, the U.S. Food and Drug Administration approved CVD 103-HgR for use in the United States. In May 2017, the American Committee on Immunization Practices (ACIP) reviewed available evidence and recommended the use of this OCV in adult international travelers, 18-64 years old, visiting areas of active cholera transmission. ${ }^{1}$ American Committee on Immunization Practices defines an area of active cholera transmission as follows: "A province, state, or other administrative subdivision within a country with endemic or epidemic cholera caused by toxigenic $V$. cholerae $\mathrm{O} 1$ and includes areas with cholera activity within the last year that are prone to recurrence of cholera epidemics; it does not include areas where only rare imported or sporadic cases have been reported."

As a result of the ACIP recommendation, the CDC began routinely reviewing global cholera surveillance data and providing data on country-specific areas of active transmission to help travelers make informed choices and health care providers make appropriate recommendations for pretravel cholera vaccination. Areas of active cholera transmission and information about the risk of cholera are provided on CDC's

\footnotetext{
*Address correspondence to Erin E. Conners, Epidemic Intelligence Service Officer Assigned to the Division of Foodborne, Waterborne, and Environmental Diseases, National Center for Emerging Zoonotic and Infectious Diseases, CDC, 1600 Clifton Rd., Atlanta, GA 30329. E-mail: ola3@cdc.gov
}

Travelers' Health Branch's country-specific destination web pages (https://wwwnc.cdc.gov/travel/destinations/list). Despite the availability of these data online, it remained unclear whether health care providers used this information to inform clinical decisions and, if so, how it is was interpreted.

\section{ASSESSING PROVIDER KNOWLEDGE}

In February 2019, CDC presented a clinical update entitled "Cholera Vaccine for Travelers" via a Clinician Outreach and Communication Activity (COCA) webinar. ${ }^{3}$ The target audience for COCA webinars are clinical professionals, and Continuing Education Credit is available to participants. Webinars are primarily advertised through the COCA e-mail subscription and through professional organizations.

The purpose of this webinar was to educate health care providers on cholera, the newly licensed OCV, ACIP guidance, and CDC cholera vaccine information resources. As part of this webinar, CDC invited participants to participate in a voluntary interactive survey. This included questions about cholera knowledge (e.g., cholera serotypes and cholera risk), cholera vaccines (e.g., type of vaccine available and administration), ACIP OCV guidance (e.g., age eligibility and recommendations), whether they use CDC resources when prescribing the OCV, and if so, how they interpret CDC's destination-specific OCV recommendations regarding areas of active cholera transmission. Questions were asked throughout the webinar and answers were given directly after each question. Responses were reported in aggregate and could not be linked to individual respondents or their occupation. Not all participants responded to the survey and questions could be skipped. The data collection activities were nonresearch and qualified for a National Childhood Vaccine Injury Act paperwork reduction act waiver. ${ }^{4}$

\section{PROVIDER KNOWLEDGE}

There were 437 webinar connections and 285 participants who registered for CME credit: 129 (45\%) nurses, 57 (20\%) physicians, 31 (11\%) pharmacists, and 68 (24\%) with other backgrounds (veterinarians, health education specialists, 
non-physicians, and public health and other professionals). In terms of general cholera knowledge, 83\% (206/249) of respondents did not know that serogroups 01 and 0139 cause cholera, and only 10\% (50/246) correctly identified that $10 \%$ of people infected with cholera have severe disease.

\section{RISK TO TRAVELERS}

Among respondents, 42\% (100/238) believed that U.S. travelers who go to countries where there is active cholera transmission are at high risk of acquiring cholera. In fact, travelers are at minimal risk of acquiring cholera if they observe safe food, water, sanitation, and handwashing recommendations while in affected countries. ${ }^{5}$ Individuals who are at increased risk of cholera include outbreak response workers or health care personnel who are treating cholera patients, those visiting friends or family, or those spending an extended period of time in a choleraaffected area.

Familiarity with this OCV was less, with only 45\% (118/263) of respondents correctly identifying oral route administration and $27 \%(69 / 252)$ correctly identifying that it is a liveattenuated vaccine. Only $18 \%$ (45/252) of respondents had previously offered the vaccine to patients; however, providers who had low knowledge of the OCV may have selfselected to participate in this webinar to learn more. We do not know what proportion of these respondents provide direct patient care.

Familiarity with the ACIP OCV guidelines was higher; $60 \%(141 / 236)$ of respondents reported knowing the ACIP recommendation for OCV for travelers going to an area of active cholera transmission in the previous 12 months, but only $47 \%(120 / 255)$ correctly identified the correct age range for eligibility.

\section{CDC TRAVELERS' HEALTH WEB SITE}

The CDC Travelers' Health Web site was used by $77 \%$ $(210 / 272)$ of respondents, either in their role as a patient or a clinician. It was also the top resource, used by $95 \%$ (247/259) of respondents, to make a decision about whether a traveler needs OCV before their trip. Other resources endorsed by participants included the CDC Yellow Book (146/259; 56\%), the WHO Web site (122/259; 47\%), and Travax (62/259; 24\%). Because the webinar respondents had low baseline knowledge on cholera and the OCV, but did use the CDC Web site for travel guidance, understanding their interpretation of this guidance on the Web site was seen as instructive for how to modify existing language for a wide audience.

Interpretation of guidance for OCV on the CDC Travelers' Health Web site varied. When provided a hypothetical scenario in which cholera was endemic in a country, 58\% $(154 / 264)$ of respondents chose to only offer OCV to adult travelers at increased risk (e.g., those providing health care). This is divergent from ACIP recommendations, which suggest offering OCV to all travelers aged 18-64 years going to an area of active transmission. When provided a scenario where one province was described as having active cholera transmission and one province was described as having their last case of cholera 7 months prior, 69\% (172/248) of respondents would offer OCV to travelers going to either province, in accordance with the ACIP guidance.

\section{UPDATES BASED ON SURVEY FINDINGS}

In response to the varied webinar results, country-specific information on the CDC Travelers' Health Web site was recently revised to include three country categories to improve guidance to providers who want to offer the OCV: 1) widespread transmission: countries where half or more of jurisdictions have reported cholera in the previous 12 months; 2) localized transmission: countries where less than half of jurisdictions have reported cholera in the previous 12 months; 3) presumed transmission: countries where there is no reliable surveillance information and cholera presence is based on expert opinion. In countries with widespread or presumed transmission, CDC recommends vaccination for travelers to any part of the country. In countries with localized transmission, CDC recommends vaccination for travelers to affected jurisdictions only.

Each country destination page lists the type of transmission with direct links to the definitions and the recommendations. For countries with localized transmission, the month of the most recent active cholera transmission reported for a given jurisdiction is available in parentheses.

ACIP bases their current recommendations on geography and the presence of cholera and not on risk factors for an individual traveler. However, during a routine pretravel consultation, each traveler should undergo a risk assessment based on their medical history, demographics, trip characteristics, and propensity to undertake behaviors that may increase their risk for illness. Considerations for providers counseling travelers to an area of active cholera transmission include whether active cholera transmission has occurred within the past 12 months, type of travel (e.g., tourism, visiting friends and family, and missionary), availability of medical care at destination, and risk factors for a poor outcome from cholera (achlorhydria, blood type $\mathrm{O}$, and medical conditions that would lead to tolerating dehydration poorly).

This survey provided insight into the knowledge and prescribing practices of U.S. providers who were interested in this OCV and allowed the CDC to adapt their communication strategy to better provide information on cholera vaccine recommendations to travelers and clinicians. Travelers and their providers should visit www.cdc.gov/travel before international travel for current information on areas of active cholera transmission, in addition to other recommendations on how to prevent cholera and stay healthy while traveling.

Received July 8, 2019. Accepted for publication October 21, 2019.

Published online January 20, 2020.

Acknowledgments: We thank Eric Mintz, Karen Wong, Laura Whitlock, Lauren Witbart, and members of the Clinician Outreach and Communication Activity at the CDC.

Disclaimer: The findings and conclusions in this report are those of the author(s) and do not necessarily represent the official position of the CDC.

Authors' addresses: Erin E. Conners, Epidemic Intelligence Service Officer assigned to the Division of Foodborne, Waterborne, and Environmental Diseases, National Center for Emerging Zoonotic and Infectious Diseases, CDC, E-mail: ola3@cdc.gov. Kristina M. Angelo and Allison T. Walker, Travelers' Health Branch, Division of Global 
Migration and Quarantine, CDC, E-mails: ydg2@cdc.gov and eie7@ cdc.gov.

\section{REFERENCES}

1. Wong KK, Burdette E, Mahon BE, Mintz ED, Ryan ET, Reingold AL, 2017. Recommendations of the advisory committee on immunization practices for use of cholera vaccine. MMWR Morb Mortal Wkly Rep 66: 482-485.

2. Levine MM, Chen WH, Kaper JB, Lock M, Danzig L, Gurwith M, 2017. PaxVax CVD 103-HgR single-dose live oral cholera vaccine. Expert Rev Vaccines 16: 197-213.
3. Centers for Disease Control and Prevention, COCA Calls/ Webinars, 2019. Clinical Update: Cholera Vaccine for Travelers. Available at: https://emergency.cdc.gov/coca/calls/2019/callinfo_ 020719.asp. Accessed May 24, 2019.

4. The Public Health Service Act National Vaccine Program, 1986. Title 42 U.S.C. 300aa-2(a)(6)-(a)(7). Available at: https:// uscode.house.gov/view.xhtml?path=/prelim@title42/chapter6A subchapter19\&edition=prelim.

5. Wong KK, Burdette E, Mintz ED, 2017. Cholera In CDC Yellow Book 2018: Health Information for International Travel. New York, NY: Oxford University Press. 